\title{
Three dimensional ultrasonography and assisted reproductive technology
}

\begin{abstract}
In the near past, two-dimensional (2D) Ultrasound was the magic key of the field of in-vitro fertilization (IVF). Nowadays, three-dimensional (3D) Ultrasound is increasingly being replacing $2 \mathrm{D}$ ultrasound for reproductive medicine. There are lots of advantages for 3D Ultrasound over the conventional 2D Ultrasound in general and in the field of reproductive medicine in particular. The 3D Ultrasound provides accurate, precise and store data of a preselected region. New techniques were introduced in 3D Ultrasound for more reproducibility and accuracy. The applicability and advantages 3D Ultrasound in assisted reproductive technologies are discussed thoroughly in this article.
\end{abstract}

Keywords: three, dimensional, ultrasound, reproductive, technology, volume, sonography, follicular development, endometrial, thickness
Volume 8 Issue I - 2019

\author{
Ahmed Altriagey,' Ayman Shehata Dawood,' \\ Gehad Sidahmed Babikir ${ }^{3}$ \\ 'Department of Obstetrics and Gynecology, Benha University, \\ Egypt \\ ${ }^{2}$ Department of Obstetrics and Gynecology, Tanta University, \\ Egypt \\ ${ }^{3}$ Department of Obstetrics and Gynaecology,Armed Forces \\ Hospitals Southern Region, Khamis Mushayt, Saudi Arabia
}

\author{
Correspondence: Ahmed Altraigey, I Department of \\ Obstetrics and Gynecology, Benha University, Benha, 43 Benha \\ Zagzig street-Mansheyet Elnoor- Benha- Qalubeya-Arab \\ Republic of Egypt ,Tel +966544854232, \\ Email ahmed.altraigy@yahoo.com
}

Received: January 14, 2019 | Published: January 30, 2019

\section{Introduction}

In the beginning of In-vitro fertilization (IVF), ovum pickup was conducted by laparoscopic oocytes retrieval followed by embryo transfer (ET) back into the uterus without any idea about the endometrial physiologic status. Also, there was no guide during the placement of the embryo transfer catheter, only dependent on clinical touch. ${ }^{1}$ Ultrasonography imaging had resolved these issues related to assisted reproduction technology (ART) procedures and helped physicians to bypass those uncertainties. The qualitative and quantitative data received from the ultrasound images are contributing to the increased success rates in ART procedures over the past two decades. ${ }^{2}$ Ultrasound importance in ART begins before stimulation protocols as Ultrasound is used in screening of pathological lesions as endometriosis, uterine anomalies, polyps, myomas and adenomyosis. All these lesions reduce the success rate of IVF cycles. Ultrasound is also used for ovarian reserve testing before treatment. Ultrasound is used for both follow-up of the response to controlled ovarian hyperstimulation, and during oocyte retrieval and embryo transfer. ${ }^{3}$

Four steps are included in 3D ultrasound examination. These steps are; acquisition of data, calculation of volume, animation of image, and storage of data for a preselected region of interest (ROI). These data had some positional information within the acquired volume dataset. The acquired 3D data can be displayed in multiple ways or analyzed in a virtual real time manner as the operators can obtain any image plane they need. ${ }^{4}$ The 3D Ultrasound provides the operator with a simultaneous display of three mutually connected, perpendicular planes with the first 3 alphabetical capital letters:

a) for sagittal plane

b) for transverse plane

c) for coronal plane

These planes maximize the available information and improve awareness of ROI. The coronal plane is unique to $3 \mathrm{D}$ ultrasound and facilitates the recognition of any surface irregularities during volume measurement. ${ }^{5}$

Volume is calculated in 3D dataset by 2 standard methods. The first method is the conventional 'full contour' method. The second method is the "rotational" method which is obtained through Virtual Organ Computer-aided Analysis (VOCALTM) that generates a 3D model of the ROI. Manual delineation of the ROI is involved in both methods and is done in the multiplanar display that shows the three characteristic perpendicular planes of 3D ultrasound. Volume measurements in 3D utilizing both methods have been proved to be more accurate than 2D measurements. Moreover, the VOCAL technique is less time consuming and allows easy rotation of the $3 \mathrm{D}$ dataset about a central axis. ${ }^{6}$

Literature review was done in PubMed, Google scholar, Academia, Mendeley, Clinical key, and Cochrane Database in the period from January, 1, 1990 till, October, 31, 2018. The specific mesh words used in search were "three dimensional ultrasound" and "assisted reproductive technology". All retrieved articles were carefully assessed and data were obtained.

\section{Three-dimensional ultrasound assessment of ovarian reserve}

Different ultrasound markers had been investigated to evaluate their role in the ovarian function prediction over the last two decades. Also, accurate assessment of volume and blood flow could be achieved by 3D ultrasound and power Doppler angiography. ${ }^{7}$ The three most common specifically used markers for ovarian reserve prediction are antral follicle count (AFC), ovarian volume, and ovarian vascularity. Investigators use conventional two-dimensional (2D) ultrasound for assessment of the ovarian morphology as well as these variables. However, the use of 3D ultrasound and power Doppler angiography as a diagnostic tool improved the predictive accuracy of ultrasonographic assessment in determining ovarian reserve. ${ }^{8}$ 


\section{Measurement of ovarian volume}

Ovarian volume is one of the markers used for evaluation of the ovarian reserve. It can be easily calculated in $2 \mathrm{D}$ images by using the formula (length $\times$ width $\times$ depth $\times \pi / 6$ ). Three-dimensional ultrasound provides a more reliable and valid method of volume calculation. The 3D ultrasound allows an accurate ovarian volume assessment and increased both intra- and inter-observer reliability. It was one of the 1 st tested markers for measuring ovarian reserve., ${ }^{9,10}$

Kyei Mensah ${ }^{11}$ used transvaginal three-dimensional (3D) ultrasound to assess the accuracy of ovarian and endometrial volume measurements in a study of 40 patients undergoing ovarian stimulation for IVF and ET. They found that Transvaginal 3D ultrasound produced highly accurate ovarian and endometrial volume measurements. ${ }^{11}$

Brett $^{12}$ on 49 infertile women, conducted transvaginal2-dimensional (2D) and 3-dimensional (3D) ultrasound in a study to assess the accuracy of ovarian reserve measurement. Two observers calculated ovarian volume. Prolate ellipsoid formula was used in 2D ultrasound examinations while virtual organ computer-aided analysis (VOCAL) was used in 3D techniques. They concluded that single measurement of ovarian volumes by $2 \mathrm{D}$ or $3 \mathrm{D}$ ultrasound was not accurate enough and two or more measurements were required. ${ }^{12}$ Rosendah 13 reported a discrepancy of approximately $30 \%$ when $2 \mathrm{D}$ Ultrasound is used to assess the pelvic organs. They stated that 2D ultrasound can't achieve a great degree of accuracy when the true ovarian volume is compared volume determined by 2D US. ${ }^{13}$

\section{Antral follicle count (AFC)}

Antral follicle count is performed commonly by $2 \mathrm{D}$ ultrasound, which helps to identify and count the number of small follicles (2$10 \mathrm{~mm}$ ) within each ovary. Assessment of the total number of antral follicles by ultrasound is considered a reliable determinant of ovarian reserve. Both 2D and 3D ultrasound could be used to count the numerous follicles in each ovary. This makes the technique tiring and the reliability and validity of counts may be uncertain. ${ }^{13-15}$

Three-dimensional ultrasound is superior to $2 \mathrm{D}$ ultrasound as it produces an image in three perpendicular planes at the same time. This gives more real orientation, as well as good data assessment. Three-dimensional ultrasound had been demonstrated to offer more reliable measurements over $2 \mathrm{D}$ imaging for AFC calculation. ${ }^{16,17}$

Antral follicle count is considered the test of choice in the assessment of diminished ovarian reserve over various ovarian reserve markers. There is a strong correlation between the number of small antral follicles and other ovarian reserve tests, such as Anti-Müllerian Hormone (AMH), giving more trust that these small follicles are the main functional ovarian reserve..$^{18}$ The use of 3D automated technique; sonography-based Automated Volume Count (SonoAVCTM) allowed precise identification of hypo-echoic, fluid filled areas within the obtained volume. Moreover, the absolute dimensions of each 3D fluid filled area could be automatically estimated by the automatic volume calculation. ${ }^{19,20}$

SonoAVC was tested in patients receiving ovarian induction and showed that the automatic measurements of both follicular diameter and volume were proved to be more reliable and more precise than $2 \mathrm{D}$ estimates for the same volume. ${ }^{19-2}$

\section{Ovarian blood flow}

More recently, 3D ultrasound has been used to capture power Doppler information, allowing the demonstration and quantification of the total blood flow within any given volume. Literature review yielded few studies testing the ability to measure ovarian blood flow and to predict ovarian response in IVF or the pregnancy occurrence in this cycles. ${ }^{21}$

Malhotra et al. correlated the peri-follicular Doppler blood flow (PFBF) and the ovarian response during in-vitro fertilization/ intracytoplasmic sperm injection (IVF/ICSI) cycles. They reported that the PFBF provided good predictor potentials for the amount of retrieved oocytes, and number of embryos. On the other hand, there was no correlations were found between PFBF and fertilization or cleavage rates. They concluded that PFBF could be used as a simple, non-invasive and independent marker for prediction of the ovarian response to gonadotropins and IVF\ICSI cycle outcome. ${ }^{22}$

Similar findings were reported in subsequent studies considering the addition of $3 \mathrm{D}$ power Doppler indices as useful parameters for ovarian reserve testing methods especially in developing multimodal simultaneous use of clinical, biochemical and 3D ultrasonographic data that makes the assessment of qualitative and quantitative aspects of ovarian reserve more accurate..$^{23,24}$

\section{Three-dimensional Ultrasound and Monitoring Treatment in ART cycles}

\section{Confirming Down-regulation}

Strict control on the hypothalamo-pituitary-ovarian axis by downregulation of the pituitary gland is the first step in both IVF/ICSI which could be achieved by continuous administration of extra physiological gonadotophin-releasing hormone agonists or antagonists. Down regulation is beneficial in preventing the growth of FSH-responsive follicles and also it ensures prevention of spontaneous ovulation, as luteinizing hormone surge is prevented ${ }^{25}$ There is no universal agreement about the best timing for ultrasound assessment of down regulation. Some researchers reported the ultrasound assessment in early follicular phase of menstrual cycle preceding IVF/ICSI ovulation induction..$^{26,27}$ On the other hand, other studies reported on assessment after laboratory evidence of down regulation. ${ }^{28}$

Jarvela $^{29}$ evaluated the ovarian changes during pituitary downregulation on 40 women undergoing IVF treatment. They concluded that no correlation is found between ovarian power Doppler signals after pituitary suppression and subsequent response to gonadotropin stimulation during IVF. They also stated that gonadotropin stimulation increases ovarian power Doppler signals due to presence of antral follicles after pituitary suppression. ${ }^{29}$

$\mathrm{Ng}$ et al. used three-dimensional (3D) power Doppler ultrasound to three ovarian parameters before and after pituitary down-regulation. The tested parameters were antral follicle count, ovarian volume and ovarian stromal blood flow. They concluded that pituitary downregulation has no effects on $\mathrm{AFC}$, ovarian volume and ovarian $3 \mathrm{D}$ power Doppler flow indices. ${ }^{28}$

Another study stated that pituitary down-regulation results in a significant reduction in ovarian volume and vascularity, but the AFC was not affected. These finding makes AFC the best predictor of ovarian response as it is not changed either measured before or after pituitary down-regulation. ${ }^{27,28}$

\section{Follicular development}

Ultrasound aids in the detection and identification of healthy follicles apart from atretic ones in both natural and IVF/ICSI cycles. ${ }^{19}$ Follicular growth is agreed as a tool to assess the adequacy of 
ovarian response to gonadotropins IVF/ICSI cycles. In most cases, 2D transvaginal ultrasound is performed to monitor follicular growth. It is well know that the supra-physiological number of follicles seemed to reduce the accuracy and ability of 2D measurements to get reliable results. This problem could be solved easily by $3 \mathrm{D}$ ultrasound techniques which give accurate identification and quantification of a precise dimensions, mean diameters, and volumes. ${ }^{30}$

Three-dimensional Ultrasound provides a new technology (SonoAVC) which measures follicular volume with reliable, welltested and automated high accuracy. It is considered more superior to the volumes obtained by either 2D device manually or those estimated by automated VOCAL. ${ }^{31}$

Rizzo et al. ${ }^{32}$ concluded that measurement of ovarian follicles by SonoAVC was found to be a very accurate and efficient and were correlated significantly to the obtained manual measurements. Also, they stated that automated volume measurements are in agreement with actual volumes of the assessed structures as well as other documented volume measurement methods. ${ }^{32}$ Moreover, the SonoAVC technique provides both reliable and highly reproducible results for different readings obtained throughout any IVF/ICSI cycle such as; $\mathrm{AFC}$, follicular volume, and follicular growth. It also assesses the embryonic volume, gestational sac, and fetal volume. Moreover, the SonoAVC measurements take less time than manual measurements. ${ }^{33}$

\section{Ovarian hyperstimulation syndrome}

Patients undergoing IVF/ICSI exhibit wide range of responses to gonadotropins. Although the majority of patients respond expectedly to the designed stimulation protocol, some women may exhibit either a poorer response to stimulation, or an exaggerated response. The exaggerated response which is known as ovarian hyperstimulation syndrome (OHSS) leads to significant morbidity and even mortality. ${ }^{34}$ Despite of the published data that studied the correlation between ovarian blood flow and its response during different controlled stimulation protocols utilized in IVF/ICSI cycles, few studies were found to address the relationship between pre-treatment ovarian blood flow and OHSS. ${ }^{35}$

Agrawal et al. assessed and compared the ovarian vascularity indices among OHSS patients by ovarian Doppler flow velocity. A statistically significant higher ovarian Doppler flow velocity in women with OHSS than controls was found. Also, their results did not show any differences in pulsatility and resistance indices between the two groups. Furthermore, they concluded that the changes in flow velocity and changes in serum and follicular fluid vascular endothelial growth factor (VEGF) concentrations were well correlated. ${ }^{36}$ In addition, other studies reported the increased ovarian stromal blood flow velocity in polycystic ovaries which is well known as one of the most important risk factors in the pathogenesis of OHSS. ${ }^{37-39}$

\section{Evaluation of endometrial receptivity}

For almost two decades, the endometrium was only assessed only using endometrial thickness measurements. Several parameters were tested to provide more valid data about the endometrial receptivity in IVF/ICSI cycles. These included endometrial patterns, endometrial/ sub endometrial blood flow indices and lastly endometrial volume measurements. These can aid in determining women with low implantation potentials. However, their predictive values and accuracy are low. During spontaneous cycles, the endometrial volume grows gradually in a specific pattern throughout the follicular phase. Then, it remains constant in the luteal phase. During follicular phase, endometrial vascularization increases till it peaks two to three days before ovulation. Endometrial volume decreases thereafter and finally increases again during mid and late luteal phase. ${ }^{40}$

\section{Assessment of endometrial thickness/volume}

Yaman et al. found that endometrial volume, in 65 women undergoing IVF program, did not show significant difference between women who conceived (Pregnancy rate was 32.3\%) and those who did not. They performed the 3D ultrasound measurements on the day of human chorionic gonadotropin (hCG) administration and found that an endometrial volume of less than $(<2.5 \mathrm{ml})$ was associated with no pregnancies. ${ }^{26}$

Also, Van Voorhis reported no correlation between pregnancy probability with endometrial thickness and volume. However, predictive values that were given by 3D power flow Doppler indices measuring endometrial perfusion could be more beneficial. ${ }^{41}$

In contrast, Merci et al., in a prospective clinical study, measured endometrial pattern, thickness, volume, and power Doppler angiography (PDA) indices of vascularization index, flow index and vascularization flow index on the day of HCG administration to evaluate their predictive power of IVF/ICSI outcome. Their results showed that 3D US PDA is useful for predicting the endometrial receptivity in IVF/ICSI cycles as endometrial volume as well as 3D power Doppler indices were statistically significant predictors of cycle outcomes. ${ }^{42}$

\section{Assessment of endometrial echogenicity}

Researches focused on the hypothesis of a possible relationship between the endometrial echogenicity degree and IVF-ET outcome. The lowest to the highest endometrial echogenicity was associated with a dramatic decline of the clinical and ongoing pregnancy rates as well as the implantation rates..$^{43}$ Järvelä et al. ${ }^{43}$ assessed and compared the sonographic characteristics of endometrium in 35 women who conceived and those with failed in IVF cycles. They studied endometrial patterns, volume and vascularization 3D PDA and concluded that the homogeneous endometrial pattern after FSH stimulation was a good prognostic sign, but for adverse outcome in IVF. The presence of a triple-line pattern after FSH stimulation as well as lower endometrial volumes seemed to be associated with better conception rates. ${ }^{43}$

\section{Assessment of endometrial/sub endometrial blood flow}

Different female reproductive functions are totally dependent on the process of angiogenesis which has a critical role in the dominant follicle development, corpus luteum formation, endometrial growth and implantation process. Thus, researches paid attention to ovarian and uterine/endometrial vascularization as a marker or predictor for predicting IVF outcomes. 3D PDA allows the study of endometrium and sub-endometrial region producing quantitative assessment of vessel density and blood flow within. ${ }^{42,44}$

There is no agreed consensus between physicians regarding the role of assessment of endometrial and sub endometrial vascularity and their results remain controversial. Ng et al. used 3D PDA in 293 women underwent their first IVF cycle to examine the predictive power of endometrial thickness, volume, vascularization index, flow index and vascularization flow index of both endometrium and sub endometrial region on two occasions, the days of HCG and ET. They found that both non-pregnant and pregnant women groups had comparable measurements on either day. They concluded that initial readings of endometrial and sub endometrial blood flow and the 
percentage changes that occurred between the measurements were not powerful predictors of pregnancy rates in IVF treatment. ${ }^{45}$

On the other hand, Wang et al., in a prospective study, assessed the role of endometrial blood supply on 182 women needed IVF-ET to predict IVF/ICSI success rates. When they detected endometrial blood flow, the woman had a higher pregnancy and implantation rates. Therefore, a strong association between blood supply and its distribution through the endometrium with the possibility for embryo implantation and development was concluded. Thus, the existence of endometrial blood flow aided the prediction of IVF-ET outcomes. ${ }^{46}$ Similarly, Choudhary et al. evaluated the role of sub endometrialendometrial blood flow assessment by Transvaginal 3D PDA and found that the presence of adequate sub endometrial-endometrial vascularity improved significantly the pregnancy outcome in IVF-ET

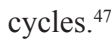

\section{Ultrasound-guided embryo transfer}

Ovarian controlled stimulation protocols, laboratory fertilization procedures and endometrial receptivity preparation are greatly improved during the years after IVF/ICSI began, but unfortunately the implantation rates did not increase in the same way, even with the transfer of good quality embryos. Thus, the area that also received extra attention was the ET technique itself. ${ }^{48}$

Even when 2D sonography started to be widely used to help in ET, the debate about the transfer distance from the fundus (TDF) was initiated. There is a controversy about the optimal placement location for embryos respecting the uterine cavity shape.$^{49} \mathrm{~A}$ new era of testing the use of 3D imaging to guide the ET catheter as it provides easily used, relatively inexpensive and probably superior images. ${ }^{50}$ The early impressions were that 3D imaging can benefit detecting the optimal embryo placement site with respect to different anatomic shapes in women. Catheter tip location in the uterine cavity was the difficulty first encountered during ET with 3D sonography. ${ }^{48}$

Sun et al. showed that 3D was associated with statistically satisfactory diagnostic accuracy, as the uterine cavity could be visualized adequately during the ET process. When they used TDF of $1-1.5 \mathrm{~cm}$ of 2D sonography, it was not accurate for embryo placement. When 3D sonography used in these patients, the embryos were actually been placed in different position. That suggested that it was inadequate to use a fixed reference point of $2 \mathrm{D}$ imaging and the precise positioning by $3 \mathrm{D}$ imaging should be implemented. ${ }^{48}$ Many researchers tried to find a solution for the dilemma whether $3 \mathrm{D}$ is superior to $2 \mathrm{D}$ ultrasound guidance during ET in improving the pregnancy rates in women undergoing ART. Their answers remained controversial as some published articles stated that there were no statistically significant differences in the pregnancy rates of women undergoing $3 \mathrm{D}$ versus $2 \mathrm{D}$ US-guided ET..$^{51,52}$ Others concluded that 3D US-guided ET into uterine cavity and catheter placement could be better and improve the fundamentals of ET technique..$^{53,54}$

\section{Conclusion}

Three-dimensional Ultrasound plays an important role in the diagnosis of women candidate for assisted reproduction. It has a great assistance in diagnosis of preexisting lesions and uterine anomalies that negatively affect implantation and pregnancy rates. Many predictive factors could be tested by 3D Ultrasound such as ovarian reserve, oocyte quality and endometrial receptivity. Moreover it is superior to 2D Ultrasound in embryo transfer techniques.

\section{Acknowledgments}

None.

\section{Conflicts of interest}

The author declares there are no conflicts of interest.

\section{References}

1. Dawood AS, Omar MK. Competing endoscopic surgeries in the era of assisted reproductive technologies: Evidence and practice. Gynecol Obstet Res Open J. 2017;4(2):20-29.

2. Porter MB. Ultrasound in assisted reproductive technology. Seminars in reproductive medicine. 2008;26(3):266-276.

3. Thalluri V, Tremellen KP. Ultrasound diagnosed adenomyosis has a negative impact on successful implantation following GnRH antagonist IVF treatment. Hum Reprod. 2012;27(12):3487-3492.

4. Jayaprakasan K, Hilwah N, Kendall NR, et al. Does 3D ultrasound offer any advantage in the pretreatment assessment of ovarian reserve and prediction of outcome after assisted reproduction treatment?. Hum Reprod. 2007;22(7):1932-1941.

5. Overbeek A, Wendel E, van den Berg MH, et al. Antral follicle counts: the application of 3D ultrasound in childhood cancer survivors. Hum Reprod. 2014;29:312-313.

6. Strizek B, Cos Sanchez T, Khalifé J, et al. Impact of operator experience on the variability of fetal lung volume estimation by 3D-ultrasound (VOCAL) and magnetic resonance imaging in fetuses with congenital diaphragmatic hernia. The Journal of Maternal-Fetal \& Neonatal Medicine. 2015;28(7):858-864

7. Wu MH, Cheng YC, Chang $\mathrm{CH}$, et al. Three-dimensional Ultrasound in Evaluation of the Ovary. Journal of Medical Ultrasound. 2012;20(3):136-141.

8. Castro EC, Borges ALF, Rezende KN, et al. Antral follicle count in predicting appropriate dose of gonadotropin in in vitro fertilization cycles. Reprod Clim. 2014;29(3):136-142.

9. Mercé LT, Gómez B, Engels V, et al. Intraobserver and interobserver reproducibility of ovarian volume, antral follicle count, and vascularity indices obtained with transvaginal 3-dimensional ultrasonography, power Doppler angiography, and the virtual organ computer-aided analysis imaging program. J Ultrasound Med. 2005; 24(9):1279-1287.

10. Kelsey TW, Wallace WH. Ovarian volume correlates strongly with the number of non-growing follicles in the human ovary. Obstetrics and gynecology international. 2012. ID 305025, 5 p.

11. Kyei Mensah A, Maconochie N, Zaidi J, et al. Transvaginal threedimensional ultrasound: reproducibility of ovarian and endometrial volume measurements. Fertil Steril. 1996;66(5):718-722.

12. Brett $\mathrm{S}, \mathrm{Bee} \mathrm{N}$, Wallace $\mathrm{WH}$, et al. Individual ovarian volumes obtained from 2-dimensional and 3-dimensional ultrasound lack precision. Reproductive biomedicine online. 2009;18(3):348-351.

13. Rosendahl M, Ernst E, Rasmussen PE, et al. True ovarian volume is underestimated by two-dimensional transvaginal ultrasound measurement. Fertil Steril. 2010;93(3):995-998.

14. Broekmans FJ, de Ziegler D, Howles CM, et al. The antral follicle count: practical recommendations for better standardization. Fertil Steril. 2010;94(3):1044-1051.

15. Practice Committee of the American Society for Reproductive Medicine. Testing and interpreting measures of ovarian reserve: a committee opinion. Fertil Steril. 2015;103(3):e9-e17.

16. Deb S, Campbell BK, Clewes JS, et al. Quantitative analysis of antral follicle number and size: a comparison of two-dimensional and automated three-dimensional ultrasound techniques. Ultrasound Obstet Gynecol. 2010;35(3):354-360.

17. Leonhardt H, Gull B, Stener Victorin E, et al. Ovarian volume and antral follicle count assessed by MRI and transvaginal ultrasonography: a methodological study. Acta Radiologica. 2014;55(2):248-256. 
18. Jayaprakasan K, Campbell B, Hopkisson J, et al. A prospective, comparative analysis of anti-Müllerian hormone, inhibin-B, and threedimensional ultrasound determinants of ovarian reserve in the prediction of poor response to controlled ovarian stimulation. Fertil Steril. 2010;93(3):855-864.

19. Singh N, Usha BR, Malik N, et al. Three-dimensional sonography-based automated volume calculation (SonoAVC) versus two-dimensional manual follicular tracking in in vitro fertilization. Int J Obstet Gynecol. 2015;131(2):166-169.

20. dos Santos Lima ML, de Paula Martins W, Nascimbem T, et al. Assessment of Ovarian Volume, Ovarian Follicle Count and Ovarian Blood Flow in Hyper Androgenic Anovulation. Advanced Topics on Three-Dimensional Ultrasound in Obstetrics and Gynecology. 2016;3(13):321-3333.

21. Hernández J, Rodríguez Fuentes A, Puopolo M, et al. Follicular Volume Predicts Oocyte Maturity: A Prospective Cohort Study Using ThreeDimensional Ultrasound and Sono AVC. Journal of Human Reproductive Sciences. 2016; 23(12):1639-1643.

22. Malhotra N, Bahadur A, Singh N, et al. Role of perifollicular Doppler blood flow in predicting cycle response in infertile women with genital tuberculosis undergoing in vitro fertilization/intracytoplasmic sperm injection. Journal of Human Reproductive Sciences. 2014;7(1):19-24.

23. Riad ON, Hak AA. Assessment of endometrial receptivity using Doppler ultrasonography in infertile women undergoing intrauterine insemination. Gynecological Endocrinology. 2014;30(1):70-73.

24. Venturella R, Gizzo S, Noventa M, et al. How age influences ovarian vascular three dimensional ultrasound parameters in a large population of Italian fertile women. Ultrasound Obstet Gynecol. 2015;46(S1):52-53.

25. Albuquerque LE, Tso LO, Saconato $\mathrm{H}$, et al. Depot versus daily administration of gonadotrophin-releasing hormone agonist protocols for pituitary down regulation in assisted reproduction cycles. The Cochrane Library. 2013;31(1):CD002808.

26. Yaman C, Ebner T, Sommergruber M, et al. Three-dimensiona endometrial volume estimation as a predictor of pituitary downregulation in an IVF-embryo transfer programme. Hum Reprod. 2000;15(8):1698-1702.

27. Jayaprakasan K, Hopkisson JF, Campbell BK, et al. Quantification of the effect of pituitary down-regulation on 3D ultrasound predictors of ovarian response. Hum Reprod. 2008;23(7):1538-1544.

28. $\mathrm{Ng} \mathrm{EH}$, Chan $\mathrm{CC}$, Yeung WS, et al. Effect of age on ovarian stromal flow measured by three-dimensional ultrasound with power Doppler in Chinese women with proven fertility. Hum Reprod. 2004;19(9):21322137.

29. Järvelä IY, Sladkevicius P, Kelly S, et al. Effect of pituitary downregulation on the ovary before in vitro fertilization as measured using three-dimensional power Doppler ultrasound. Fertil Steril. 2003;79(5):1129-1135.

30. Murtinger M, Zech NH. 3D Ultrasound for Follicle Monitoring in ART. In: Stadtmauer L, Tur Kaspa I, editors. Ultrasound Imaging in Reproductive Medicine. New York, NY: Springer; 2014. 360 p.

31. Raine Fenning N, Jayaprakasan K, Clewes J, et al. Sono AVC: a novel method of automatic volume calculation. Ultrasound Obstet Gynecol. 2008;31(6):691-696

32. Rizzo G, Capponi A, Pietrolucci ME, et al. Sonographic automated volume count (Sono AVC) in volume measurement of fetal fluid-filled structures: comparison with Virtual Organ Computer-aided Analysis (VOCAL). Ultrasound Obstet Gynecol. 2008;32(1):111-112.

33. Ata B, Tulandi T. Ultrasound automated volume calculation in reproduction and in pregnancy. Fertil Steril. 2011;95(7):2163-2170.

34. Humaidan P, Quartarolo J, Papanikolaou EG. Preventing ovarian hyperstimulation syndrome: guidance for the clinician. Fertil Steril. 2010;94(2):389-400.
35. Jayaprakasan K, Deb S, Batcha M, et al. The cohort of antral follicles measuring 2-6 $\mathrm{mm}$ reflects the quantitative status of ovarian reserve as assessed by serum levels of anti-Müllerian hormone and response to controlled ovarian stimulation. Fertil Steril. 2010;94(5):1775-1781.

36. Agrawal R, Conway G, Sladkevicius P, et al. Serum vascular endothelial growth factor and Doppler blood flow velocities in in vitro fertilization: relevance to ovarian hyperstimulation syndrome and polycystic ovaries. Fertil Steril. 1998;70(4):651-658.

37. Jayaprakasan K, Jayaprakasan R, Al Hasie HA, et al. Can quantitative three-dimensional power Doppler angiography be used to predict ovarian hyperstimulation syndrome? Ultrasound Obstet Gynecol. 2009;33(3):583-91.

38. Smith LP. Ultrasound and Ovarian Hyperstimulation Syndrome. In Ultrasound Imaging in Reproductive Medicine. New York: Springer; 2014. Pp. 303-313.

39. Pascual MA, Graupera B, Hereter L, et al. Assessment of ovarian vascularization in the polycystic ovary by three-dimensional power Doppler ultrasonography. Gynecol Endocrinol. 2008;24(11):631-636.

40. Žáčková T, Järvelä IY, Marděšic T. The Role of $3 D$ Ultrasound in Assessment of Endometrial Receptivity and Follicular Vascularity to Predict the Quality Oocyte. Ultrasound Imaging-Medical Applications. In: Oleg Minin, editor. 2011. 19 p.

41. Van Voorhis BJ. Clinical practice. In: vitro fertilization. $N$ Engl J Med. 2007;356(4):379-386.

42. Mercé LT, Barco MJ, Bau S, Troyano J. Are endometrial parameters by three-dimensional ultrasound and power Doppler angiography related to in vitro fertilization/embryo transfer outcome?. Fertil Steril. 2008;89(1):111-117.

43. Järvelä IY, Sladkevicius P, Kelly S, et al. Evaluation of endometria receptivity during in-vitro fertilization using three-dimensional power Doppler ultrasound. Ultrasound Obstet Gynecol. 2005;26(7):765-769.

44. Ng EH, Chan CC, Tang OS, et al. The role of endometrial and sub endometrial blood flows measured by three-dimensional power Doppler ultrasound in the prediction of pregnancy during IVF treatment. Hum Reprod. 2005;21(1):164-170.

45. Ng EH, Chan CC, Tang OS, et al. Changes in endometrial and sub endometrial blood flow in IVF. Reproductive biomedicine online. 2009;18(2):269-275

46. Wang L, Qiao J, Li R, et al. Role of endometrial blood flow assessment with color Doppler energy in predicting pregnancy outcome of IVF-ET cycles. Reproductive Biology and Endocrinology. 2010;8(1):122.

47. Choudhary M, Chowdhary J, Swarankar ML, et al. Predictive value of subendometrial-endometrial blood flow assessment by transvaginal 3D power Doppler on the day of HCG on clinical outcome of IVF cycles. International Journal of Research in Medical Sciences. 2017;3(11):3114 3118.

48. Sun Y, Fang L, Su Y, Guo Y. Advantages of 3Dimensional Sonography in Embryo Transfer. J Ultrasound Med. 2009;28(5):573-578.

49. Lambers MJ, Dogan E, Lens JW, et al. The position of transferred air bubbles after embryo transfer is related to pregnancy rate. Fertil Steril. 2007;88(1):68-73.

50. Letterie GS. Three dimensional ultrasound guided embryo transfer: a preliminary study. Am J Obstet Gynecol. 2005;192(6):1983-1987.

51. Saravelos SH, Kong GW, Chung JP, et al. A prospective randomized controlled trial of 3D versus 2D ultrasound-guided embryo transfer in women undergoing ART treatment. Hum Reprod. 2016;31(10):22552260 .

52. Kosmas IP, Janssens R, De Munck L, et al. Ultrasound-guided embryo transfer does not offer any benefit in clinical outcome: a randomized controlled trial. Hum Reprod. 2007;22(5):1327-1334. 
53. Fang L, Sun Y, Su Y, et al. Advantages of 3-Dimensional Sonography in Embryo Transfer. Journal of Ultrasound in Medicine. 2009;28(5):573578 .
54. Gergely RZ, DeUgarte CM, Danzer H, et al. Three dimensional/four dimensional ultrasound-guided embryo transfers using the maximal implantation potential point. Fertil Steril. 2005;84(2):500-503. 